\title{
Comparison of Model Predictive Control (MPC) and Linear-Quadratic Gaussian (LQG) Algorithm for Electrohydraulic Steering Control System
}

\author{
Alexander Mitov ${ }^{1, *}$, Jordan Kralev ${ }^{2}$, Tsonyo Slavov ${ }^{2}$ and Ilcho Angelov ${ }^{1}$ \\ ${ }^{1}$ Technical University of Sofia, Dept. of Hydroaerodynamics and Hydraulic Machines, Kliment \\ Ohridski blvd. №8, Bulgaria \\ ${ }^{2}$ Technical University of Sofia, Dept. of Systems and Control, Kliment Ohridski blvd. 8, Bulgaria
}

\begin{abstract}
The paper compares the performance of two embedded controllers applied in electrohydraulic steering systems - model predictive controller (MPC) and linear-quadratic Gaussian (LQG) controller with Kalman filtering for state estimation. Both controllers are designed on the basis of single input multiple output "black box" model obtained via identification approach. The controllers are implemented into industrial logic controller for mobile applications and their workability is experimentally checked with a laboratory model of a steering system for non-road mobile machinery. The results corresponding to investigation of performance of the closed-loop system are presented.
\end{abstract}

\section{Introduction}

From theoretical point of view, LQG and MPC are similar because of the analytical form of the cost function which is quadratic weighting plant state and the control signal. However, the approaches used for the minimization are quite distinct. The design of LQG usually takes integral of the cost from initial time to infinity (so-called infinite time case) which leads to fast converging solution of the differential Ricatti equation allowing to formulate linear state feedback controller. Then the state is estimated with the help of Kalman filter assuming white noise disturbance acting in the state and output equations. The MPC design assumes finite time quadratic cost function and incorporates amplitude and rate constraints for the control and state signals [1]. The solution, which minimizes the cost function, is obtained during each sampling interval by iterative procedure with respect to the control action sequence for the whole prediction horizon. However, only the first value of that sequence is actually applied as a current control action, because in the next sampling period the optimization is repeated. During the optimization, the system model is used to predict the state and output value.

These two quadratic approaches to the control of electrohydraulic steering unit are not investigated much in literature and the main question is whether we can achieve some considerable benefit in terms of tracking performance or robustness by employing more

\footnotetext{
*Corresponding author: a mitov@tu-sofia.bg
} 
computationally intensive MPC strategy or more theoretically guaranteed approach with LQG.

The main purpose of this article is a comparative analysis of the performance for the closed-loop systems with two embedded controllers applied in electrohydraulic steering systems - model predictive controller (MPC) and linear-quadratic Gaussian (LQG) controller with Kalman filtering for state estimation. For the both system respective statespace mathematical models are identified to facilitate the controller synthesis. To investigate the performance of the closed-loop system several control indexes are determinate. The comparative analysis of time and frequency domain properties of controllers are performed. A number of experimental tests describes synergistic effect between the two subsystems electrohydraulic and embedded control systems.

\section{Hardware layout}

\subsection{Power steering system description}

The embedded MPC and LQG controllers are subjected for a power steering laboratory test rig which is based on EHSU type OSPEC200 LSRM. The laboratory system is developed respect to technical data sheet from producer of mobile machinery equipment [2]. The circuit diagram of hydraulic system of the test system is in detail described in [3].

\subsection{CAN communication system}

The main proposition of this article is a developed real-time system for study of MPC and LQG algorithms for control of electrohydraulic steering device (Fig. 1). The data acquisition and physical signal manipulation are done by using a controller type MC012-022 [1]. The plant measured output signals are the proportional spool position, flow rate and cylinder piston position. The control action from the controller is a pulse width modulated voltage applied to the PVE electrohydraulic block. The communication between the PLC and the Simulink ${ }^{\circledR}$ real-time simulation model, which is deployed on the conventional workstation, is performed via CAN communication channel. The main control system signals are sent to workstation for real-time visualization. Therefore, the computational possibilities of the PLC are extended with those of the dedicated workstation. Such architecture requires that the rate of transmitted data must be significantly higher than the control system sampling time. The communication initiates from the controller. It sends a data packages with $10 \mathrm{~ms}$ period respect to the control system sampling interval. So within that period the message is transmitted to the Simulink ${ }^{\circledR}$ model. Then it is starting on the workstation for a time $\tau_{1}$, evaluating control signal for a time $\tau_{2}$ and a response message is send back to the microcontroller for a time $\tau_{3}$. The time critical element in the so described communication protocol is the time for processing a single iteration on workstation. However if we assume that the workstation is fast enough then the duration of communication is $\tau_{1}+\tau_{2}+\tau_{3}<0.01$. Thus we obtain the system which function in real-time. To interfaces with the CG150 USB/CAN communication cable converter, we design a specialized communication block, which uses MATLAB $^{\circledR}$ Vehicle Network Toolbox. In it, components for CAN communication adapted for various vendors are included. The block developed receives CAN messages in a blocking mode. Hence the execution of the simulation model is blocked until the message synchronized with the $10 \mathrm{~ms}$ time frame arrives. Then the model blocks are executed for one step and when the execution of the communication block is in order it blocks simulation again till arrival of the consequent CAN message from the MC012-022 
microcontroller. Since the workstation, computational possibilities can be extended significantly, the proposed architecture allows implementation of more complicated and computationally demanding controllers like MPC.

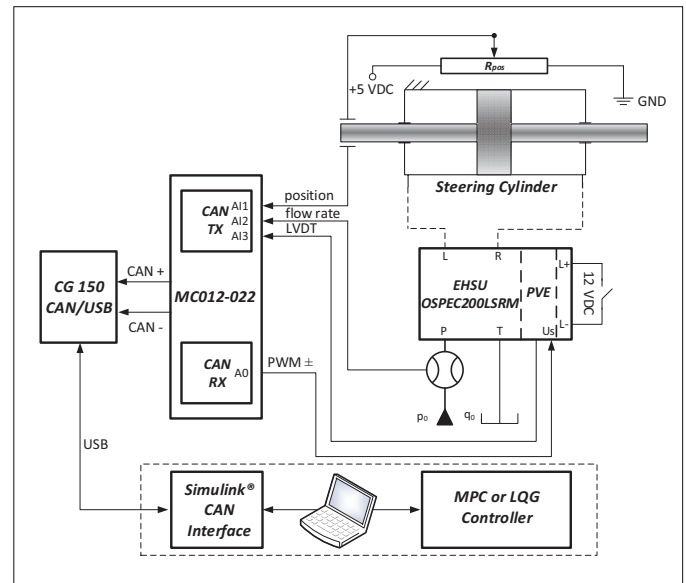

Fig. 1. Extended computational system for real-time implementation of designed controllers.

\section{System model}

To record input-output, data open loop identification experiment is performed (Fig.2). The sample rate is $40 \mathrm{~Hz}$.

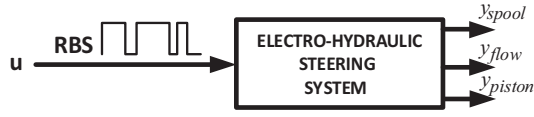

Fig. 2. Block scheme of open-loop identification experiment.

The system used in identification procedure [4] is composed of microprocessor, hydraulic steering device, steering servo cylinder and piston position sensor. The data set for plant parameters estimation is depicted in Fig. 3 where the manipulated variable is shown in $\mathrm{mV}$ for increased quantization.

The obtained model takes the state space form

$$
\begin{aligned}
& x(k+1)=A x(k)+B u(k)+K e(k) \\
& y(k)=C x(k)+D u(k)+e(k),
\end{aligned}
$$

where $\quad x=\left(\begin{array}{lll}x_{1} & x_{2} & x_{3}\end{array}\right)^{T}$ is the state vector, $u$ is the control signal, $y=\left(\begin{array}{lll}y_{\text {spool }} & y_{\text {flow }} & y_{\text {piston }}\end{array}\right)^{T}$ is the output signal and $e(k)=\left(\begin{array}{lll}e_{1} & e_{2} & e_{3}\end{array}\right)^{T}$ is the vector of residual errors [5]. The matrices model (1)-(2) are 
and covariance matrix of residual error is

$$
\begin{aligned}
A & =\left(\begin{array}{ccc}
-1.05 & 0.19 & 6.27 \\
-0.04 & 1 & 0.198 \\
-0.371 & 0.033 & 2.044
\end{array}\right), B=10^{-3}\left(\begin{array}{c}
-0.37 \\
-0.034 \\
-0.23
\end{array}\right), \\
C & =\left(\begin{array}{ccc}
-31 & 0.67 & -6.58 \\
-0.584 & 0.007 & -0.25 \\
0.059 & -2.019 & 0.125
\end{array}\right), D=10^{-3}\left(\begin{array}{c}
0.117 \\
0.014 \\
0.0021
\end{array}\right), \\
K & =\left(\begin{array}{ccc}
-0.025 & -0.023 & -0.022 \\
-0.002 & 0.0066 & -0.429 \\
-0.007 & -0.01 & 0.0033
\end{array}\right)
\end{aligned}
$$

$$
M\left(e e^{T}\right)=10^{3}\left(\begin{array}{ccc}
7.92 & 0.034 & 0.011 \\
0.034 & 0.03 & 0.001 \\
0.011 & 0.001 & 0.003
\end{array}\right) .
$$

\section{MPC and LQG Controller Design}

\subsection{MPC design}

For the state space model (1)-(2), the state variables can be estimated by the Kalman filter

$$
\hat{x}(k+1)=A \hat{x}(k)+B u(k)+L(k)(y(k)-C \hat{x}(k)-D u(k)),
$$

where the Kalman gain is obtained by

$$
L(k)=P(k \mid k-1) C^{T}\left(C P(k \mid k-1) C^{T}+R\right)^{-1},
$$

where $P(k \mid k-1)$ is the covariance matrix of the estimation error $x(k)-x_{h a t}(k \mid k-1)$. $P(k \mid k-1)$ is the solution of the Ricatti equation

$$
\begin{aligned}
& P(k+1 \mid k)=A P(k \mid k-1) A^{T}+K Q K^{T}- \\
& A P(k \mid k-1) C^{T}\left(C P(k \mid k-1) C^{T}+R\right)^{-1} C P(k \mid k-1) A^{T} .
\end{aligned}
$$

The MPC problem [6] is defined by the objective function

$$
\vec{u}_{t}=\operatorname{Arg} \min _{\vec{u}_{t}} \sum_{k=0}^{N-1} q\left(x_{t+k}, u_{t+k}\right),
$$

which have to be minimized with respect to the control signal for the prediction horizon of $N$ sampling intervals from the system future subject to initial state $x_{t}=x(t)$, system state equation (1), fixed control horizon $M<N$, state and control amplitude constraints and rate constraints. For prediction of system behavior, the system model is used [5]. The main benefit is explicit account for constraints in the controller design.

$$
\vec{u}_{t}=\left(u_{t}, u_{t+1}, \ldots, u_{t+N-1}\right)
$$

with $u(t+i-1)=u(t+m-1)$ for $i>m$. When the optimal sequence of control signal is determined only the first control action $u_{t}$ is implemented to the plant. In the next sample interval the optimization procedure is repeated again and new input sequence is generated. In most applications, the aim of control system is that the controller should keep plant outputs at or near specified desired values. Thus the sample cost becomes

$$
q\left(x_{i}, u_{i}\right)=w_{1} y_{1, i}^{2}+w_{2} y_{2, i}^{2}+w_{3}\left(r-y_{3, i}\right)^{2}
$$


where the LVDT, flow rate and cylinder position error are taken into account. The MPC solves a quadratic programming problem at each sampling interval and the solution is the control signal which is applied to the plant only for the current sampling interval. It should be noted that to compute the cost function the controller needs to calculate the predicted values of all output variables which depends on the future input sequence and on the model initial state [7]. The optimization problem is converted to the general $Q P$ form by the MPC $Q P$ solver

$$
0.5 x^{T} H z+f^{T} x, A x \leq b,
$$

where $\mathrm{x}$ and $\mathrm{z}$ are the decision variables, $H$ is the Hessian matrix, $A$ is a matrix of linear constraint coefficients, and $b$ and $f$ are vectors. To solve the $Q P$ problem, the $K W I K$ algorithm is used in blocks from Simulink ${ }^{\circledR}$ Model Predictive control toolbox, which requires $H>0$. The initial guess of the algorithm is the unconstrained solution. If it satisfies the constraints, it is the optimal $Q P$ solution. Otherwise this means that at least one of the linear inequality constraints must be satisfied as an equality - active constraint set satisfying the standard optimality conditions. In the following control steps the solution from the previous step becomes an initial guess for the next. The constraints on the control signal are set to $\pm 5000 \mathrm{mV}$. The output weights of MPC cost function are set for $w_{1}=0, w_{2}=0.8$ and $w_{3}=1$.

\subsection{LQG controller design}

The block diagram of control system based on LQR controller and Kalman filter is shown in Figure 3. The LQR with integral action is designed, which provides good reference tracking [8]. The synthesis is performed for the estimated model (3)-(4). In this model the additional state is introduced. It is discrete time integral of position error

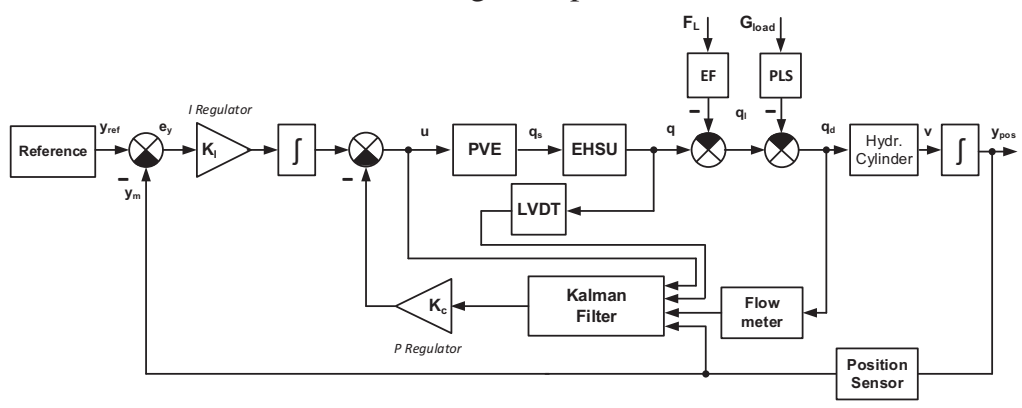

Fig. 3. Structure of the LQG closed-loop system.

$$
x_{i}(k+1)=x_{i}(k)+T_{s} e_{v}(k)=x_{i}(k)+T_{s}\left(y_{\text {ref }}(k)-y_{\text {pos }}(k)\right),
$$

where is the reference. Thus, combining the deterministic parts of equation (3) and (4) with equation (12) one obtains the augmented system

$$
\begin{aligned}
& \bar{x}(k+1)=\bar{A} \bar{x}(k)+\bar{B} u(k)+\bar{G} y_{\text {ref }}(k), \\
& y(k)=\bar{C} \bar{x}(k),
\end{aligned}
$$

where $\bar{x}(k)=\left|\begin{array}{c}x(k) \\ x_{i}(k)\end{array}\right|, \bar{A}=\left|\begin{array}{cc}A & 0_{4 \times 1} \\ -T_{S} C_{3} & 1\end{array}\right|, \bar{B}=\left|\begin{array}{c}B \\ 0\end{array}\right|, \bar{C}=\left|\begin{array}{cc}C & 0_{3 \times 1}\end{array}\right|, \bar{G}=\left|\begin{array}{c}0_{3 \times 1} \\ T_{S}\end{array}\right|$ and $C_{3}$ is the third row of matrix $C$.

The controller algorithm is selected as

$$
u(k)=-\bar{K} \bar{x}(k), \bar{K}=\left[\begin{array}{ll}
K_{c} & -K_{i}
\end{array}\right],
$$


$K_{c}$ is matrix gain of state feedback term and $K_{i}$ is the integral component. The optimal matrix $\bar{K}$ is obtained by minimization of

$$
J(u)=\sum_{k=0}^{\infty} \bar{x}^{T}(k) Q \bar{x}(k)+u^{T}(k) R u(k),
$$

Where $Q$ and $R$ are positive definite matrices chosen to provide acceptable performance of closed-loop system. The optimal matrix $\bar{K}$ is determined by [4]

$$
\bar{K}=\left(R+\bar{B}^{T} P \bar{B}\right)^{-1} \bar{B}^{T} P \bar{A},
$$

where $P$ is the positive definite solution of the discrete-time matrix algebraic Riccati equation

$$
\bar{A}^{T} P \bar{A}-P-\bar{A}^{T} P \bar{B}\left(R+\bar{B}^{T} P \bar{B}\right)^{-1} \bar{B}^{T} P \bar{A}+Q=0 .
$$

The controller matrices $K_{c}=\left[\begin{array}{lll}9.26 & -56.18 & -45.19\end{array}\right]$ and $K_{i}=9.96$ are obtained for $Q=\left[\begin{array}{cc}10^{3} & 0_{1 \times 3} \\ 0_{3 \times 1} & 500 C^{T} C\end{array}\right]$ and $R=100$.

The state variables $x(k)$ is not accessible, so the optimal control law (4) is formed as

$$
u(k)=-K_{c} \hat{x}(k)+K_{i} x_{i}(k),
$$

where $\hat{x}(k)$ is estimate of $x(k)$. It is obtained by discrete time Kalman filter (5) and (6).

\section{Comparison analysis of the results with designed controllers}

The analytical comparison between MPC and LQG controllers is difficult because the LQG closed system is linear, however the predictive controller is nonlinear by its design and so is the closed-loop system with it. However, we know that the real plant dynamics is only slightly nonlinear so we should be able to linearize the closed loop system with the predictive controller.

In order to do that, we convey an identification experiment with the MPC controller and the plant model. The input excitation to the MPC closed-loop system is reference signal which is composed of step function to $100 \mathrm{~mm}$ and a frequency sweep from 0.005 to $0.3 \mathrm{~Hz}$ additively introduced in the reference channel after $30 \mathrm{sec}$, when the cylinder position reaches the target. The final time for the frequency sweep is set to $200 \mathrm{sec}$ and its amplitude is $10 \mathrm{~mm}$. The output signal for identification is cylinder piston position obtained from simulation of the nonlinear system containing the electrohydraulic steering system model resampled at $10 \mathrm{~ms}$ and the designed MPC. We have estimated a $6^{\text {th }}$ order ARX model of the form

$$
A\left(q^{-1}\right) y_{\text {piston }}(t)=B\left(q^{-1}\right) r(t)+e(t),
$$

where the polynomials $A$ and $B$ are estimated from the recorded 20000 samples of data to

$$
\begin{gathered}
A\left(q^{-1}\right)=1-2.13 q^{-1}+q^{-2}+0.03 q^{-3}+0.16 q^{-4}+0.19 q^{-5}-0.26 q^{-6}, \\
B\left(q^{-1}\right)=\left(0.28+7.43 q^{-1}-14.42 q^{-2}+5.92 q^{-3}+3.11 q^{-4}-2.19 q^{-5}\right) \times 10^{-5} .
\end{gathered}
$$

The model achieves $100 \%$ FIT to the identification data when used as a predictor and $76 \%$ FIT when used as simulator. The final prediction error is $7.12 \times 10^{-6}$ and the mean square error is $7.11 \times 10^{-6}$. Estimated model can be used to compare the MPC and LQG closed-loop systems in frequency domain. The comparison is presented in Fig.4. 


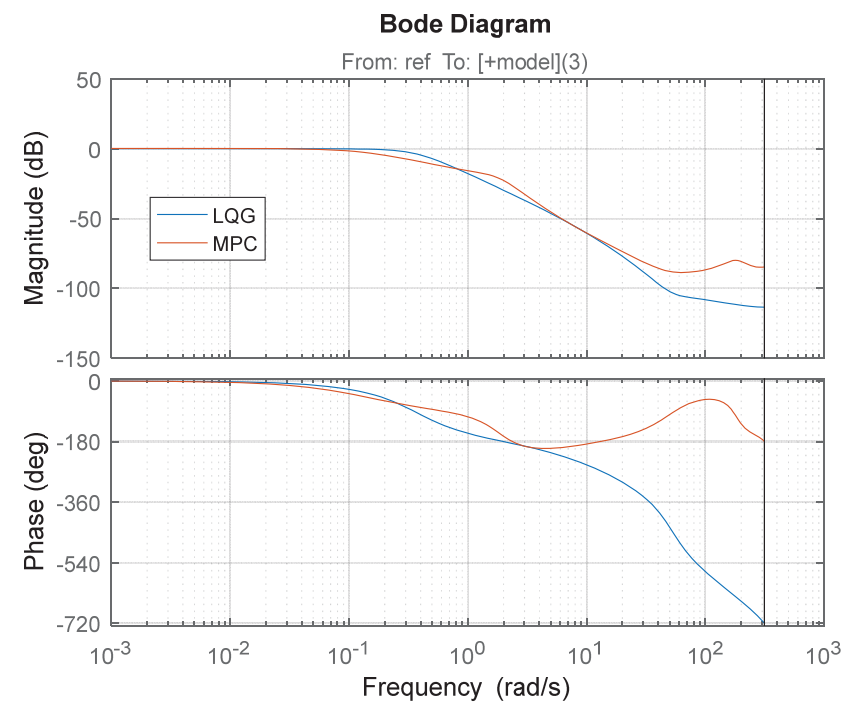

Fig. 4. Complementary sensitivity function.

As can be seen both system have analogical amplitude frequency response in the low and middle frequency ranges. The bandwidth of both systems are comparable, the system with the LQG has slightly larger bandwidth, which we may assume that can be compensated with additional tuning of the controller. However, the MPC demonstrate a higher gain in the high frequency range, which makes it more sensitive to measurement noise.

Fig. 5 compares the sensitivity of the closed-loop system with both controllers. The differences here are more pronounced in comparison to previous figure. The LQG demonstrate a clearly better disturbance attenuation - it scales down output disturbances more than 1000 times at $0.0001 \mathrm{rad} / \mathrm{s}$. However, the MPC closed-loop system is more sensitive to output disturbances by attenuating their amplitudes no more than 50 times. This can be a strong argument to prefer LQG design before the MPC. Of course, here we again have to say that MPC specification can be alleviated in such a way to increase the low frequency disturbance attenuation by introduction of respective disturbance model.

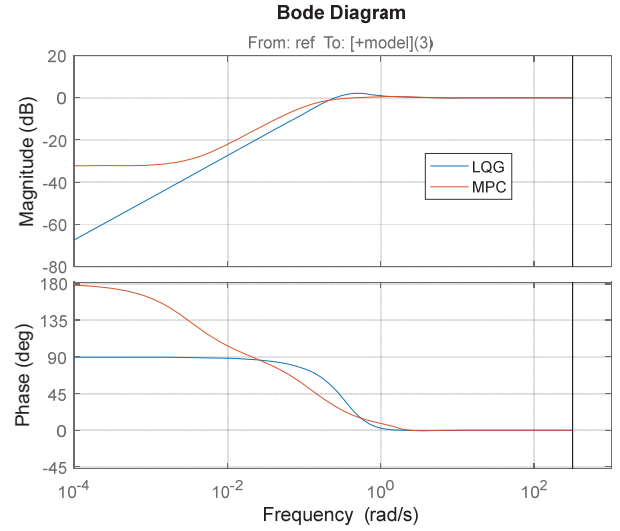

Fig. 5. Sensitivity function of the closed-loop error with respect to output disturbance.

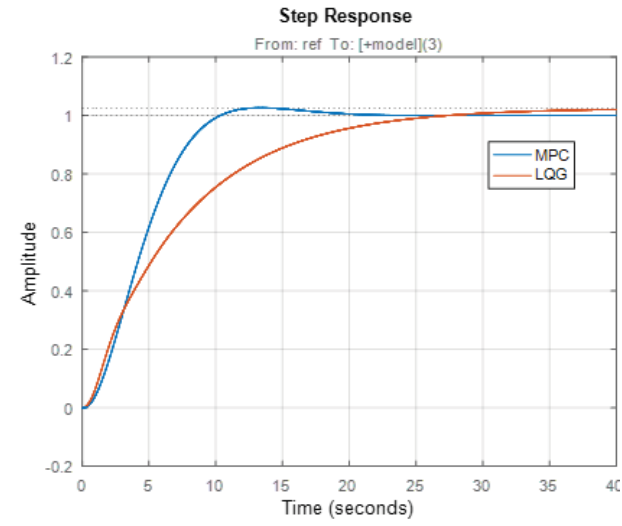

Fig. 6. Step response of the closed-loop system.

The step responses of the closed-loop system with LQG and MPC controllers are compared on the Fig.6. In addition to better attenuation of output disturbances, the LQG is 
clearly faster as a response, achieving its steady state level around $10 \mathrm{sec}$. The MPC step response is around twice slower - $20 \mathrm{sec}$. However, we observe a small overshooting for the LQG which may be undesirable for tracking performance. But we've decided to investigate this more during the experiments with the electrohydraulic steering system test bench.

\subsection{Implementation of designed controllers}

The experiments with MPC and LQG controllers are performed by the specialized Simulink ${ }^{\circledR}$ model depicted in the Fig.7. The communication on the CAN channel between MC012-022 and the workstation is supported through MATLAB ${ }^{\circledR}$ Function block. This block also guarantees that the model will run in a blocking mode of operation. The designed control algorithms is realized in state-space block for LQG and in the MPC Controller block for the MPC.

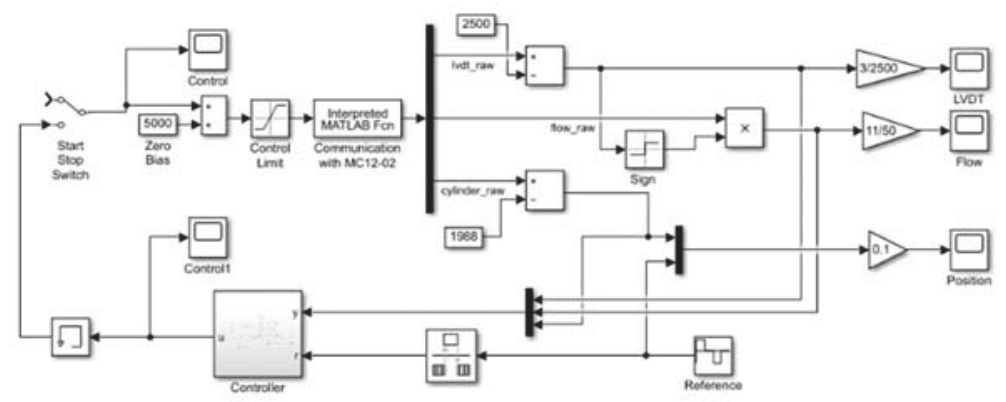

Fig.7. Specialized Simulink ${ }^{\circledR}$ model for controller’s implementation.

\subsection{Comparison of experimental results}

Tracking performance of the closed-loop system with the MPC and LQG controller is compared in Fig.8. Both controller reach the reference for an approximately identical time. However, there is clear pronounced overshoot of the system with the MPC controller in the first two steps from the reference signal. Generally, the tracking accuracy of the MPC system is worse because we can see prolonged period of non-zero tracking error. The LQG controller achieves zero tracking error for approximately $5 \mathrm{sec}$ which is uniform in both directions of piston translation. Also the LQG controller reaches the target in an aperiodic transient which is indicative for better stability margin.

The control signal from two controllers are depicted in the Fig. 9. The control signals generated from MPC and LQG controller share similar features as maximal amplitude and steady state. However, we may notice that the control signal of the LQG controller is smoother and less oscillatory than the MPC signal.

Figure 10 presents the position of the internal spool valve, which is responsible for directing the flow through the steering cylinder. Both controllers react with a maximal valve opening in the beginning of the transient when the actual positon is far from the desired piston positon. However, when the tracking error is small the reaction of LQG and MPC controller is radically different. The MPC controller move the spool fast in the opposite direction to compensate for the positon overshooting. After that the spool is opened once more in the initial direction to compensate the second period of the tracking error oscillation. In addition there are observed high frequency oscillations in the spool positon with the MPC controller which are not problematic for the spool mechanics but can create flow rate instability which is evident in the Fig.8. The reaction of the spool position for the closed-loop system with the 
LQG controller is aperiodic. Instead of bumping in the opposite direction the LQG controller begin to close the spool valve when the tracking error becomes small enough which dampens the accumulated kinetic energy in the system. This allows for more accurate positioning of the cylinder while reaching the target.

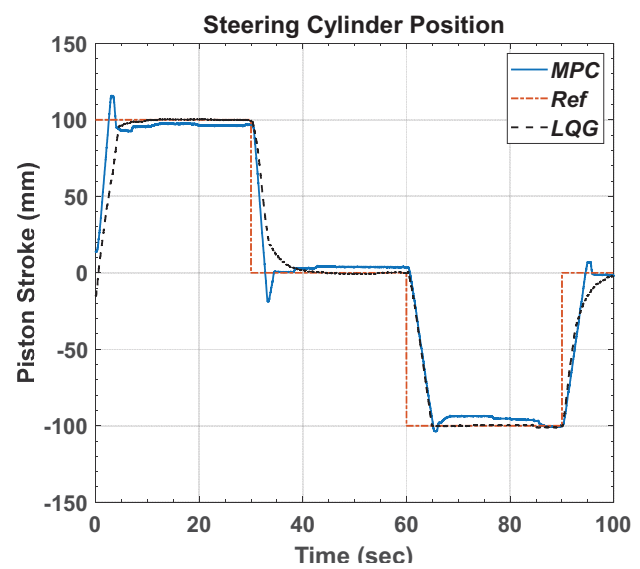

Fig.8. Comparison of measured cylinder piston transient response.

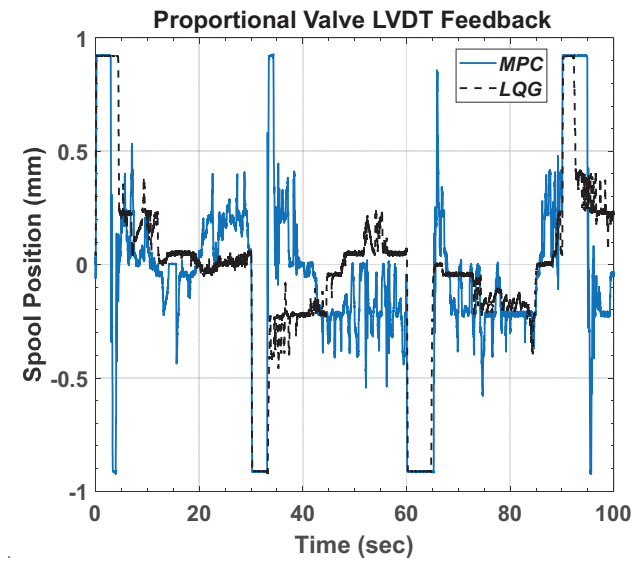

Fig.10. Comparison of measured spool position.

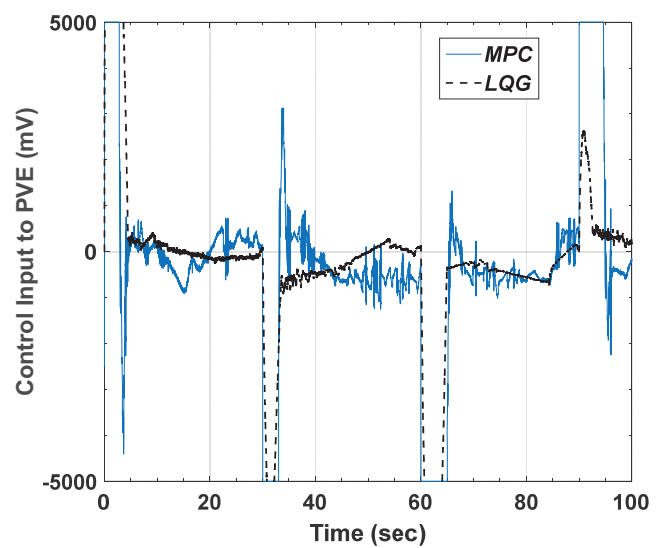

Fig.9. Comparison of measured control signal.

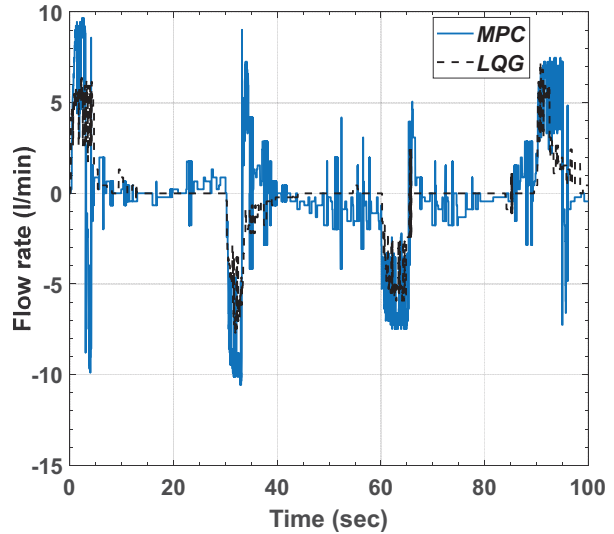

Fig.11. Comparison of measured flow rate.

Figure 11 compares the flow rate for the MPC and the LQG controller measured at the inlet port of the electrohydraulic steering unit in $1 / \mathrm{min}$. As can be seen the flow rate for the closed-loop system with the MPC controller is more oscillatory which is not very acceptable for the hydraulic systems since the transients of flow direction changes are related to extreme pressure events which are not advantageous for the system [9]. The flow rate of the closedloop system with the LQG controller is practically zero at steady state which is result of the improved accuracy of the positional loop. When the desired position is achieved no flow is needed for a correction. The maximal amplitudes of the flow rate achieved with the both control algorithms are comparable, however the LQG amplitude is smaller. Generally, the LQG controller requires less energy to achieve the desired position. 


\section{Conclusions}

The results achieved in this articles show that for an electrohydraulic steering systems which can be described well enough with a linear time-invariant models the linear-quadratic Gaussian controllers should be preferred before the model predictive controller. There are several reasons which are in favour of LQG implementation. First is considerable more compact and less computationally intensive software algorithms which can be executed on most of the modern specialized PLC controllers for mobile applications. Second the performance of the LQG controller in the experimental evaluation conveyed in this paper proved better than the MPC in terms of relative stability and accuracy. Of course we don't exclude the possibility of additional tuning of MPC parameters in order to reach a better performance. However, such tuning may require considerable more time in comparison with the LQG controller tuning. In addition, proving the stability and robustness of MPC algorithms will require a lot more effort than for LQG, where feasibility of controller is guaranteed in a large extend during the synthesis. Practical implementation of the MPC as an embedded algorithm require a lot more software modules including quadratic programming algorithms, full scale model simulation, memory for the predicted signals. Alternative to this is implementation of explicit MPC control where a piecewise linear mapping is defined of the state space facilitating feedback control. However, achieving an effective approximation of such mapping requires additional computational time and have to be justified by the complexity of the system dynamics.

\section{References}

1. E. Kayacan, H. Ramon, W. Saeys, Distributed nonlinear model predictive control of an autonomous tractor-trailer system, Mechatronics, 24(8), 926-933, (2014).

2. Danfoss, "OSPE Steering Valve”, Technical Information, 11068682, November, (2016).

3. Il. Angelov, Al. Mitov, Test Bench for Experimental Research and Identification of Electrohydraulic Steering Units, International Fluid Power Conference, 10thIFK 2016, Dresden, Germany, (2016).

4. Al. Mitov, J. Kralev, Ts. Slavov, Il. Angelov, SIMO System Identification of Transfer Function Model for Electrohydraulic Power Steering, 16th International Conference on Electrical Machines, Drives and Power Systems (ELMA), Bulgaria, (2019).

5. L. Ljung, System Identification: Theory for the User. Prentice-Hall, Inc., Englewood Cliffs, NJ, 2nd edition, ISBN 978-0136566953, (1999).

6. Al. Mitov, Al., J. Kralev, Ts. Slavov, Il. Angelov, Model Predictive Control Design for Electro-hydraulic Power Steering Application, The 9th Mediterranean Conference on Embedded Computing - MECO'2020, ISBN 978-1-5386-5683-9, Montenegro, (2020).

7. G. Raffo, G. Gomes, J. Normey-Rico, C. Kelber, L. Becker, “A Predictive Controller for Autonomous Vehicle Path Tracking", IEEE Transactions on Intelligent Transportation Systems, 10(1), 92-102, (2009).

8. K. Zhou, J.C. Doyle, etc., Robust and Optimal Control, Prentice Hall International, Inc., Upper Saddle River, NJ, ISBN 0-13-456567-3, (1996).

9. P. Tomov, B. Aprahamyan, K. Petrov, P. Andreev, P. Petkov. Experimental survey and analysis on the impact of the commutation process on a positive displacement pump driven by an asynchronous electric motor, 16th International Conference on Electrical Machines, Drives and Power Systems (ELMA), ISBN 978-1-5090-6690-2, Bulgaria, (2019). 